\title{
COMPLETELY REGULAR GROWTH SOLUTIONS OF SECOND ORDER COMPLEX LINEAR DIFFERENTIAL EQUATIONS
}

\author{
Janne Heittokangas, Ilpo Laine, Kazuya Tohge and Zhi-Tao Wen \\ University of Eastern Finland, Department of Physics and Mathematics \\ P.O. Box 111, 80101 Joensuu, Finland; janne.heittokangas@uef.fi \\ University of Eastern Finland, Department of Physics and Mathematics \\ P.O. Box 111, 80101 Joensuu, Finland; ilpo.laine@uef.fi \\ Kanazawa University, College of Science and Engineering \\ Kakuma-machi, Kanazawa 920-1192, Japan; tohge@se.kanazawa-u.ac.jp \\ Taiyuan University of Technology, Department of Mathematics \\ Yingze West Street, No. 79, Taiyuan 030024, China; zhitaowen@gmail.com
}

\begin{abstract}
If $A(z)$ and $B(z)$ are transcendental entire functions, then all solutions of the differential equation $f^{\prime \prime}+A(z) f^{\prime}+B(z) f=0$ are entire and typically of infinite order. Simple examples show that finite order solutions are also possible. Assuming that $A(z)$ and $B(z)$ are of completely regular growth, Gol'dberg-Ostrovskii-Petrenko asked whether all solutions of finite order are of completely regular growth also. This problem remains unsolved, but several aspects of the problem are addressed. Exponential polynomials form an important subclass of functions of completely regular growth, and they are known to always satisfy certain linear differential equations. Hence cases where the coefficients and/or the solutions are exponential polynomials are also discussed.
\end{abstract}

\section{Introduction}

Supposing that $A(z)$ and $B(z)$ are entire functions, it is well known that all solutions of

$$
f^{\prime \prime}+A(z) f^{\prime}+B(z) f=0
$$

are entire functions also. Moreover, Wittich has proved that all solutions of (1.1) are of finite order of growth if and only if both $A(z)$ and $B(z)$ are polynomials [24]. Hence, if at least one of $A(z)$ or $B(z)$ is transcendental, there must be solutions of infinite order. In general, if (1.1) possesses a nontrivial solution of finite order of growth, then

$$
T(r, B) \leq T(r, A)+O(\log r)
$$

by [6, Theorem 2]. Here $T(r, g)=m(r, g)+N(r, g)$ denotes the standard Nevanlinna characteristic of $g$. Finite order solutions are indeed possible:

Example 1.1. If $A(z)=e^{z}$ and $B(z)=e^{z}-1$, then $f(z)=e^{-z}$ solves (1.1).

doi:10.5186/aasfm.2015.4057

2010 Mathematics Subject Classification: Primary 34M05; Secondary 30D35.

Key words: Completely regular growth, complex differential equation, exponential polynomial, Phragmén-Lindelöf indicator function.

Acknowledgement: This research was financially supported by the Academy of Finland \#268009, the Chinese Scholarship Council (CSC), and the Japan Society for the Promotion of Science Grant-in-Aid for Scientific Research (C) \#25400131. 
We shall be dealing with cases where the coefficients and/or solutions of (1.1) are of completely regular growth, or c.r.g. for short. For the definition of c.r.g., recall first that the Phragmén-Lindelöf indicator function of an entire $g$ of finite order $\rho=\rho(g)>0$ is

$$
h_{g}(\theta)=\limsup _{r \rightarrow \infty} r^{-\rho} \log \left|g\left(r e^{i \theta}\right)\right|, \quad \theta \in[-\pi, \pi) .
$$

For example, if $g(z)=\exp \left(w z^{n}\right)$, where $w \in \mathbf{C} \backslash\{0\}$ and $n$ is a positive integer, then $h_{g}(\theta)=\Re\left(w e^{i n \theta}\right)$. It is clear that $h_{g}$ is $2 \pi$-periodic in general. If $g$ is of finite type, then $h_{g}$ is continuous, and the maximal value of $h_{g}$ equals

$$
\tau_{M}(g)=\limsup _{r \rightarrow \infty} r^{-\rho} \log M(r, g),
$$

which is the $M$-type of $g$ [1, Chpt. 5]. Following [17, p. 6], we say that an entire function $g$ is of c.r.g. if it is of finite type with respect to its order $\rho=\rho(g) \in(0, \infty)$, and if

$$
r^{-\rho} \log \left|g\left(r e^{i \theta}\right)\right| \rightarrow h_{g}(\theta)
$$

as $r \rightarrow \infty$ outside of a possible exceptional set of zero upper density this set being the same for each value of $\theta$. The upper and lower densities of a set $E \subset[0, \infty)$ are given, respectively, by

$$
\overline{\operatorname{dens}}(E)=\limsup _{r \rightarrow \infty} \frac{\int_{1}^{r} \chi_{E}(t) d t}{r-1} \quad \text { and } \quad \underline{\operatorname{dens}}(E)=\liminf _{r \rightarrow \infty} \frac{\int_{1}^{r} \chi_{E}(t) d t}{r-1},
$$

where $\chi_{E}$ denotes the characteristic function of the set $E$.

Example 1.2. If $g(z)=e^{z}$, then $\log \left|g\left(r e^{i \theta}\right)\right|=r \cos \theta$, while if $g$ is either of the trigonometric functions $\sin z=\left(e^{i z}-e^{-i z}\right) /(2 i)$ or $\cos z=\left(e^{i z}+e^{-i z}\right) / 2$, then $\log \left|g\left(r e^{i \theta}\right)\right|=r|\sin \theta|+o(1)$. In all three cases, $g$ is of c.r.g.

The research in this paper is motivated by the following problem of Gol'dberg, Ostrovskii and Petrenko [8, p. 300], or GOP-problem for short, originally stated for linear differential equations of arbitrary order.

GOP-problem. Suppose that $A(z)$ and $B(z)$ are entire functions of completely regular growth. If (1.1) possesses an entire solution $f$ of finite order of growth, then is it true that $f$ is of completely regular growth?

As noted in [8, p. 300] and proved in [16, p. 110], if $A(z)$ and $B(z)$ are polynomials, then $f$ is of c.r.g. If $A(z)$ and $B(z)$ are not of c.r.g., then $f$ is not necessarily of c.r.g. Indeed, Gol'dberg has shown [8, p. 300] that if $f$ is any entire function with at most simple zeros, then $f$ is a solution of some differential equation of the form (1.1) with entire coefficients $A(z), B(z)$. It is of course essential that the solution should be transcendental, for polynomial solutions are possible even in the case when both of the coefficients are transcendental: If $A(z)=z e^{z}$ and $B(z)=-e^{z}$, then $f(z)=z$ solves (1.1).

Exponential polynomials form an important subclass of functions of c.r.g. Here an exponential polynomial of order $n$ is an entire function of the form

$$
g(z)=P_{1}(z) e^{Q_{1}(z)}+\cdots+P_{l}(z) e^{Q_{l}(z)},
$$

where $P_{j}$ 's and $Q_{j}$ 's are polynomials in $z$ with $\max _{j}\left\{\operatorname{deg}\left(Q_{j}\right)\right\}=n$.

Lemma 1.3. Any exponential polynomial $g$ of the form (1.3) is of c.r.g. 
Proof. As described in [18], $g$ can be written in the normalized form

$$
g(z)=H_{0}(z)+H_{1}(z) e^{w_{1} z^{n}}+\cdots+H_{m}(z) e^{w_{m} z^{n}},
$$

where $m \leq k, H_{j}$ 's are either exponential polynomials of order $\leq n-1$ or ordinary polynomials in $z$, and $w_{j}$ 's are pairwise different nonzero complex constants called the leading coefficients of $g$. It follows from the proof of [18, Satz 4], see also [19, p. 462], that

$$
h_{g}(\theta)=\lim _{r \rightarrow \infty} r^{-n} \log \left|g\left(r e^{i \theta}\right)\right|
$$

with finitely many possible exceptional values of $\theta$ on $[-\pi, \pi)$. Hence an exponential polynomial is of c.r.g. on every ray with at most finitely many exceptions. By [17, Theorem 1.3.4] or [14, p. 140] it follows that exceptional rays are not possible, and hence $g$ is of c.r.g.

In addition to being of c.r.g., exponential polynomials $g$ of the form (1.3) satisfy a linear differential equation of order

$$
k \leq \sum_{j=1}^{m}\left(1+\operatorname{deg}\left(P_{j}\right)\right) n^{l-j}
$$

with polynomial coefficients [22]. Here $n=\max _{j}\left\{\operatorname{deg}\left(Q_{j}\right)\right\}$. Hence the multiplicity of zeros of $g$ cannot exceed $k-1$.

The GOP-problem is complicated and remains unsolved. Assuming the coefficients $A(z), B(z)$ to be exponential polynomials seems like a natural place to start. We also consider the cases where $f$ is an exponential polynomial or an exponential of an exponential polynomial.

A few things from [18] need to be reviewed. The convex hull $\operatorname{co}(W)$ of a finite set $W \subset \mathbf{C}$ is the intersection of finitely many closed half-planes each containing $W$. Hence $\operatorname{co}(W)$ is either a compact polygon or a line segment. We denote the perimeter of $\operatorname{co}(W)$ by $C(\operatorname{co}(W))$. If $\operatorname{co}(W)$ is a line segment, then $C(\operatorname{co}(W))$ equals twice the length of this line segment. Related to the leading coefficients in (1.4), we denote $W=\left\{\overline{w_{1}}, \ldots, \overline{w_{m}}\right\}$ and $W_{0}=W \cup\{0\}$.

Theorem A. [18] Let $g$ be given by (1.4). Then

$$
T(r, g)=C\left(\operatorname{co}\left(W_{0}\right)\right) \frac{r^{n}}{2 \pi}+o\left(r^{n}\right) .
$$

If $H_{0}(z) \not \equiv 0$, then

$$
m\left(r, \frac{1}{g}\right)=o\left(r^{n}\right)
$$

while if $H_{0}(z) \equiv 0$, then

$$
N\left(r, \frac{1}{g}\right)=C(\operatorname{co}(W)) \frac{r^{n}}{2 \pi}+o\left(r^{n}\right) .
$$

The remainder of this paper is organized as follows. In Section 2 we consider infinite order solutions, and hence set aside cases where the solutions of (1.1) are not of c.r.g. Section 3 is devoted to considering exponential polynomials as solutions since they are typical examples of functions of c.r.g., and since they always solve some linear differential equation. Given that the solutions of (1.1) are typically of infinite order, we illustrate in Section 4 (among other things) that it is not that 
uncommon for an exponential of an exponential polynomial to be a solution. The paper is supplemented with a variety of examples which illustrate the sharpness of the main results.
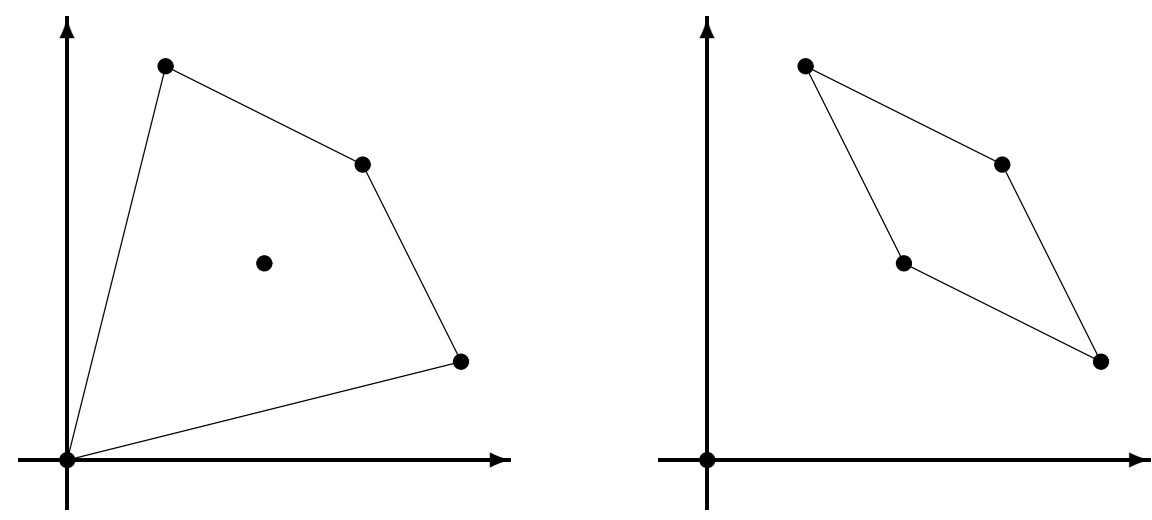

Figure 1. The convex hull of finitely many points. The perimeter can be affected if the origin is included/excluded.

\section{Infinite order solutions}

As noted in [6], if $A(z)$ and $B(z)$ are entire such that $\rho(A)<\rho(B)$, then $(1.2)$ implies that all solutions $f \not \equiv 0$ of $(1.1)$ are of infinite order. More specifically, if $\rho(A)=\rho(B)>0$ but the $T$-types satisfy $\tau(A)<\tau(B)$, where

$$
\tau(g)=\limsup _{r \rightarrow \infty} r^{-\rho(g)} \log T(r, g),
$$

then $\rho(f)=\infty$ by (1.2). In both cases the solutions are not of c.r.g., while in the case $\tau(A)=\tau(B)$ this is possible, see Example 1.1. We proceed to state a slightly more delicate result in terms of the Phragmén-Lindelöf indicator function. It is possible that this result is essentially known at least to specialists, but the authors haven't been able to find an actual statement anywhere.

Theorem 2.1. Let $A(z)$ and $B(z)$ be entire such that $\rho(A)=\rho(B) \in(0, \infty)$, and assume that both are of finite type. If (1.1) possesses a solution $f \neq \equiv 0$ of finite order, then

$$
h_{B}(\theta) \leq \max \left\{0, h_{A}(\theta)\right\}, \quad \theta \in[-\pi, \pi) .
$$

In particular, if there exists a $\theta_{0} \in[-\pi, \pi)$ such that $\max \left\{0, h_{A}\left(\theta_{0}\right)\right\}<h_{B}\left(\theta_{0}\right)$, then all solutions of (1.1) are of infinite order.

Proof. Write (1.1) as

$$
-B(z)=A(z) \frac{f^{\prime}}{f}+\frac{f^{\prime \prime}}{f}
$$

and then use a logarithmic derivative estimate [5, Corollary 1] to see that (2.1) holds for almost every $\theta$. Since $A(z)$ and $B(z)$ are of finite type, the indicator functions are continuous, and so (2.1) holds for every $\theta$. The remaining assertion is a trivial consequence of (2.1). 
Example 2.2. If $P(z)$ is a nonconstant polynomial, then all nontrivial solutions of the differential equation $f^{\prime \prime}+e^{P(z)} f^{\prime}+e^{-P(z)} f=0$ are of infinite order by Theorem 2.1. This result is observed in [6, p. 418] but the proof is different from ours.

If $A(z)$ and $B(z)$ are entire such that $\rho(B)<\rho(A) \leq 1 / 2$, then all solutions of (1.1) are of infinite order [6], [10]. For any integer $n \geq 1$, finite order solutions may occur in the case

$$
0<\rho(B)<\rho(A)=n,
$$

see Examples 1 and 2 in [6]. Next we will show that if $A(z)$ is an exponential polynomial and if $B(z)$ is entire such that (2.2) holds, then all solutions of (1.1) are of infinite order.

Theorem 2.3. Let $A(z)$ be an exponential polynomial, and let $B(z)$ be entire such that (2.2) holds for some $n \geq 1$. Suppose that the set $E_{1}=\{\theta \in$ $\left.[-\pi, \pi): h_{A}(\theta) h_{B}(\theta)=0\right\}$ is of Lebesgue measure zero. Then all solutions of (1.1) are of infinite order.

We will rely on the following result due to Kwon [11, Theorem 2] on more than one occasions. The proof is given for the convenience of the reader.

Lemma 2.4. Let $A(z)$ and $B(z)$ be entire functions such that $\rho(A)>\rho(B)$. Then every solution $f \not \equiv 0$ of (1.1) satisfies $\rho(f) \geq \rho(A)$.

Proof. We may suppose that $\rho(f)<\infty$, for otherwise the assertion is trivially true. Equation (1.1) yields

$$
|A(z)| \leq\left|\frac{f^{\prime \prime}(z)}{f^{\prime}(z)}\right|+|B(z)| \frac{f(z)}{f^{\prime}(z)} \mid .
$$

Thus

$$
\begin{aligned}
T(r, A) & =m(r, A) \leq m(r, B)+m\left(r, f / f^{\prime}\right)+O(\log r) \\
& \leq T(r, B)+T\left(r, f^{\prime} / f\right)+O(\log r) \\
& \leq T(r, B)+3 T(r, f)+O(\log r) .
\end{aligned}
$$

The assertion then follows by the assumption $\rho(A)>\rho(B)$.

Proof of Theorem 2.3. Suppose on the contrary to the claim that $\rho(f)<\infty$. By Lemma 2.4 we know that $\rho(f) \geq \rho(A)=n \geq 1$. Hence, by [5, Corollary 1], for every $\varepsilon>0$ there exists a set $E_{2} \subset[-\pi, \pi)$ of measure zero such that if $\theta \notin E_{2}$, then there is a constant $R_{0}=R_{0}(\theta)>1$ such that for all $z$ satisfying $\arg z=\theta$ and $|z| \geq R_{0}$, we have

$$
\left|\frac{f^{\prime \prime}(z)}{f^{\prime}(z)}\right| \leq|z|^{\rho(f)-1+\varepsilon} .
$$

Let $E_{3} \subset[-\pi, \pi)$ be the finite exceptional set related to (1.5), with $A(z)$ in place of $g$. Now $E=E_{1} \cup E_{2} \cup E_{3}$ is of linear measure zero. We choose $\theta \in[-\pi, \pi) \backslash E$ and divide the proof into three cases as follows.

Case 1: $h_{A}(\theta)>0$. Suppose that $\left|f^{\prime}(z)\right|$ is unbounded on $\arg z=\theta$. Then, by $[6$, Lemma 4], there exists an infinite sequence of points $z_{n}=r_{n} e^{i \theta}$ tending to infinity such that $f^{\prime}\left(z_{n}\right) \rightarrow \infty$ and

$$
\left|f\left(z_{n}\right) / f^{\prime}\left(z_{n}\right)\right| \leq(1+o(1))\left|z_{n}\right|
$$


as $n \rightarrow \infty$. Using (2.2), (2.4), (2.5), (1.5) and the assumption $h_{A}(\theta)>0$ on (2.3), we arrive at a contradiction. Hence $\left|f^{\prime}(z)\right|$ must be bounded on the $\operatorname{ray} \arg z=\theta$. Integrating along the line segment $[0, z]$ and using the standard formula

$$
f(z)=f(0)+\int_{0}^{z} f^{\prime}(\zeta) d \zeta
$$

we obtain $|f(z)|=O(|z|)$ as $|z| \rightarrow \infty$ such that $\arg z=\theta$.

Case 2: $h_{A}(\theta)<0$ and $h_{B}(\theta)<0$. Suppose that $\left|f^{\prime \prime}(z)\right|$ is unbounded on $\arg z=\theta$. Then, by [13, Lemma 3.1], there exists an infinite sequence of points $z_{n}=r_{n} e^{i \theta}$ tending to infinity such that $f^{\prime \prime}\left(z_{n}\right) \rightarrow \infty$ and

$$
\left|\frac{f^{(j)}\left(z_{n}\right)}{f^{\prime \prime}\left(z_{n}\right)}\right| \leq \frac{1}{2-j}(1+o(1))\left|z_{n}\right|^{2-j}, \quad j=0,1,
$$

as $n \rightarrow \infty$. Equation (1.1) yields

$$
1 \leq|A(z)|\left|\frac{f^{\prime}(z)}{f^{\prime \prime}(z)}\right|+|B(z)| \frac{f(z)}{f^{\prime \prime}(z)} \mid .
$$

Using (2.7) and the assumptions $h_{A}(\theta)<0$ and $h_{B}(\theta)<0$, we arrive at a contradiction. Hence $\left|f^{\prime \prime}(z)\right|$ must be bounded on the ray $\arg z=\theta$. This gives us $|f(z)|=O\left(|z|^{2}\right)$ as $|z| \rightarrow \infty$ such that $\arg z=\theta$.

Case 3: $h_{A}(\theta)<0$ and $h_{B}(\theta)>0$. We arrive at an immediate contradiction by the proof of Theorem 2.1.

So far we have that either $\rho(f)=\infty$ or $\rho(f)<\infty$ and $|f(z)|=O\left(|z|^{2}\right)$ as $|z| \rightarrow \infty$ such that $\arg z=\theta \notin E$, where $E$ has measure zero. In the latter case we can make use of the standard Phragmén-Lindelöf method to conclude that $|f(z)|=O\left(|z|^{2}\right)$ in the whole plane. This means that $f$ is a polynomial, which is a contradiction. Hence $\rho(f)=\infty$ must hold.

The assumption on the set $E_{1}$ in Theorem 2.3 involves both of the coefficients $A(z)$ and $B(z)$. Alternatively it is enough to assume that the set $E=\{\theta \in$ $\left.[-\pi, \pi): h_{A}(\theta) \leq 0\right\}$, which only involves the coefficient $A(z)$, has measure zero to conclude that all solutions of (1.1) are of infinite order. This is a special case of the following theorem.

Theorem 2.5. Let $A(z)$ be an entire function of c.r.g., and let $B(z)$ be any entire function such that $\rho(B)<\rho(A)$. Define $E=\left\{\theta \in[-\pi, \pi): h_{A}(\theta) \leq 0\right\}$. Then every solution $f \not \equiv 0$ of (1.1) satisfies

$$
\rho(f) \geq \max \left\{\rho(A),(21 \sqrt{m(E)})^{-1}-1\right\}
$$

where $\rho(f)=\infty$ if $m(E)=0$. Here $m(E)$ is the Lebesgue measure of $E$.

Alongside the linear densities of $E \subset[0, \infty)$ defined in Section 1, we will also make use of the corresponding logarithmic densities

$$
\overline{\log \operatorname{dens}}(E)=\limsup _{r \rightarrow \infty} \frac{\int_{1}^{r} \frac{\chi_{E}(t)}{t} d t}{\log r} \text { and } \underline{\log \operatorname{dens}}(E)=\liminf _{r \rightarrow \infty} \frac{\int_{1}^{r} \frac{\chi_{E}(t)}{t} d t}{\log r} .
$$

The following inequalities can be found in [20, p. 121]:

$$
0 \leq \underline{\operatorname{dens}}(E) \leq \underline{\log \operatorname{dens}}(E) \leq \overline{\log \operatorname{dens}}(E) \leq \overline{\operatorname{dens}}(E) \leq 1 .
$$


Proof of Theorem 2.5. The inequality $\rho(f) \geq \rho(A)$ follows by Lemma 2.4. Hence we may suppose that $(21 \sqrt{m(E)})^{-1}-1>\rho(A)$. If $m(E)=0$, we consider this to be trivially true. Suppose on the contrary to the claim that (1.1) has a solution $f \not \equiv 0$ for which $\rho(f)<(21 \sqrt{m(E)})^{-1}-1$. In particular, $f$ is of finite order of growth.

Case 1: $m(E)>0$. Let $\varepsilon>0$ be fixed and small enough so that

$$
\rho(f)<\frac{1-\varepsilon}{7(3+\varepsilon) \sqrt{m(E)}}-1<\frac{1}{21 \sqrt{m(E)}}-1 .
$$

Since $\rho(f)<\infty$, we may use [5, Corollary 2] to deduce that there exists a set $F_{1} \subset(1, \infty)$ of finite logarithmic measure such that

$$
\left|\frac{f^{\prime \prime}(z)}{f(z)}\right| \leq|z|^{2(\rho(f)-1+\varepsilon)}, \quad|z| \notin F_{1} \cup[0,1] .
$$

It is clear that $\overline{\log \operatorname{dens}}\left(F_{1}\right)=0$.

The proof of $[6$, Theorem 6$]$ shows that $f$ must have infinitely many zeros, or, in fact, the exponent of convergence of zeros of $f$ must be positive. This allows us to use the following special case of [15, Theorem 1], applied to the solution $f$ : For $r>0$ and for the constant $\varepsilon>0$ in (2.9), let

$$
U_{r}=\left\{\theta \in[0,2 \pi]: r\left|\frac{f^{\prime}\left(r e^{i \theta}\right)}{f\left(r e^{i \theta}\right)}\right| \geq \varepsilon n(r, 1 / f)\right\} .
$$

Then there exists a set $F_{2} \subset[1, \infty)$ with $\underline{\log \operatorname{dens}}\left(F_{2}\right) \geq \varepsilon /(3+\varepsilon)$ such that

$$
m\left(U_{r}\right)>\left(\frac{1-\varepsilon}{7(3+\varepsilon)(\rho(f)+1)}\right)^{2}, \quad r \in F_{2} .
$$

The assumption (2.9) now guarantees that $m\left(U_{r}\right)>m(E)$, or $m\left(U_{r} \backslash E\right)>0$.

Since $A(z)$ is of completely regular growth, it follows by [17, Theorem 1.2.1] that

$$
\log |A(z)|=r^{\rho(A)} h_{A}(\theta)+o\left(r^{\rho(A)}\right)
$$

for $z=r e^{i \theta}$ outside of a possible $C_{0}$-set $D \subset \mathbf{C}$. Such a set can be covered by a system of Euclidean discs $D\left(a_{n}, r_{n}\right)$ such that

$$
\lim _{r \rightarrow \infty} r^{-1} \sum_{\left|a_{n}\right| \leq r} r_{n}=0
$$

see $\left[17\right.$, p. 7]. Let $F_{3}$ be the projection of $D$ onto the non-negative real axis. Then $F_{3}$ is covered by the intervals $\left(\left|a_{n}\right|-r_{n},\left|a_{n}\right|+r_{n}\right)$ of length $2 r_{n}$. Consequently, $\overline{\log \operatorname{dens}}\left(F_{3}\right) \leq \overline{\operatorname{dens}}\left(F_{3}\right)=0$ by $(2.8)$ and $(2.12)$.

Define $F=F_{2} \backslash\left(F_{1} \cup F_{3}\right)$. Then $\underline{\log \operatorname{dens}}(F) \geq \varepsilon /(3+\varepsilon)$. Write (1.1) in the form

$$
-A(z) \frac{f^{\prime}}{f}=B(z)+\frac{f^{\prime \prime}}{f} .
$$

If $z=r e^{i \theta}$ is such that $r \in F$ and $\theta \in U_{r} \backslash E$, we conclude that

$$
\varepsilon r^{-1} n(r, 1 / f) \exp \left(r^{\rho(A)} h_{A}(\theta)+o\left(r^{\rho(A)}\right)\right) \leq M(r, B)+r^{2(\rho(f)-1+\varepsilon)},
$$

where $h_{A}(\theta)>0$. Since $m\left(U_{r} \backslash E\right)$ is bounded away from zero for all $r$, we may let $r \rightarrow \infty$. This contradicts the assumption $\rho(B)<\rho(A)$. 
Case 2: $m(E)=0$. Now $\rho(f)<\infty$, so that (2.10) is still valid. As for the set $U_{r}$, we choose

$$
U_{r}=\left\{\theta \in[0,2 \pi]: 2 r\left|\frac{f^{\prime}\left(r e^{i \theta}\right)}{f\left(r e^{i \theta}\right)}\right| \geq n(r, 1 / f)\right\} .
$$

Then there exists a set $F_{2} \subset[1, \infty)$ with $\underline{\log \operatorname{dens}}\left(F_{2}\right) \geq 1 / 4$ such that

$$
m\left(U_{r}\right)>\left(\frac{1}{56(\rho(f)+1)}\right)^{2}, \quad r \in F_{2} .
$$

It is clear that $m\left(U_{r}\right)>0$, or $m\left(U_{r} \backslash E\right)>0$. The rest of the proof follows that of Case 1.

Example 2.6. (1) If $A(z)=P(z) e^{z}+Q(z) e^{-z}+R(z)$, where $P, Q, R$ are polynomials, then $h_{A}( \pm \pi / 2)=0$ and $h_{A}(\theta)>0$ otherwise, so that $m(E)=0$ in Theorem 2.5. Hence, if $B(z)$ is any entire function with $\rho(B)<1$, then all solutions of (1.1) are of infinite order.

(2) The assumption $\rho(A)>\rho(B)$ is necessary in Theorem 2.5: The function $f(z)=e^{z}+1$ solves $f^{\prime \prime}+\left(e^{-z}-e^{z}-1\right) f^{\prime}+\left(e^{z}-1\right) f=0$ although $m(E)=0$.

\section{Exponential polynomial solutions}

Assuming that the coefficients $A(z)$ and $B(z)$ are exponential polynomials, finite order solutions of (1.1) may exist, see Examples 1.1 and 3.1.

Example 3.1. [7, Example 3.6] If $A(z)=e^{2 z}+e^{z}+1$ and $B(z)=e^{z}$, then $f(z)=e^{-z}+1$ solves (1.1).

In general, exponential polynomial solutions may exist even if $A(z)$ and $B(z)$ are not exponential polynomials [6, p. 416]: If $H(z)$ is any entire function and $k \in \mathbf{N}$, then $f(z)=\exp \left(z^{k}\right)$ solves (1.1), where

$$
A(z)=H(z)-k z^{k-1} \quad \text { and } \quad B(z)=-\left(k(k-1) z^{k-2}+k z^{k-1} H(z)\right) .
$$

Conversely, even if the coefficients $A(z)$ and $B(z)$ are exponential polynomials, then a finite-order solution of (1.1) does not have to be an exponential polynomial. This can be seen by [6, Example 1], where one may choose $Q(z)=z^{2}$ and $B(z)=e^{z}$.

The following example reveals various cases in which (1.1) has an exponential polynomial solution.

Example 3.2. (1) It is possible that $A(z)$ and $f$ are exponential polynomials of equal order, while $B(z)$ is an ordinary polynomial in $z$. Indeed, following [6, Example 1], let $B(z) \not \equiv 0$, and let $A(z)=-1-B(z)\left(1+e^{-z}\right)$. Then $f(z)=e^{z}+1$ solves (1.1).

(2) It is possible that $\rho(f)=\rho(A)$ and either $\rho(A)>\rho(B)$ or $\rho(A)=\rho(B)$ when $f$ has $m \geq 2$ leading coefficients: If $A(z)=-11-6 e^{-z}$ and $B(z)=18$, then $f(z)=1+3 e^{z}+2 e^{2 z}$ solves (1.1). If $A(z)=e^{z}+(3 / 2) e^{-z}$ and $B(z)=-3-2 e^{z}$, then $f(z)=1+2 e^{z}+2 e^{2 z}$ solves (1.1).

(3) Modifying [6, Example 2], we show that any integer order $\rho(f) \geq 2$ is possible for an exponential polynomial solution $f$. Let $P$ be a polynomial with degree $\geq 2$ such that $P^{\prime}$ has only simple zeros, and that $P^{\prime}(z) \neq 0$ whenever $\exp (-P(z))=1$. Let $Q$ be an entire function having the interpolation property $Q(z)=P^{\prime \prime}(z) /\left(e^{-P(z)}-1\right)$ whenever $P^{\prime}(z)=0$. Since only finitely many values need to be interpolated, we may 
choose $Q$ to be Lagrange's interpolation polynomial satisfying $Q \not \equiv 0$ and $\operatorname{deg}(Q) \leq$ $\operatorname{deg}(P)-2$. Set

$$
\begin{aligned}
& B(z)=Q(z)+P^{\prime}(z) e^{R(z)} \\
& A(z)=-\left(f^{\prime \prime}+B(z) f\right) / f^{\prime} \\
& f(z)=e^{P(z)}-1,
\end{aligned}
$$

where $R$ is an arbitrary polynomial with $\operatorname{deg}(R) \leq \operatorname{deg}(P)-1$. If $f^{\prime}(z)=0$, then $P^{\prime}(z)=0, B(z)=P^{\prime \prime}(z) /\left(e^{-P(z)}-1\right)$, and hence $A(z)$ is entire. Now (1.1) is satisfied, $B(z)$ and $f$ are exponential polynomials, while

$$
A(z)=\left(\frac{Q(z)}{P^{\prime}(z)}+e^{R(z)}\right)\left(e^{-P(z)}-1\right)-\frac{P^{\prime \prime}(z)}{P^{\prime}(z)}-P^{\prime}(z)
$$

is essentially an exponential polynomial in the sense that it has rational coefficients with removable poles. In particular, $A(z)$ is of c.r.g. Moreover,

$$
\rho(B)=\operatorname{deg}(R)<\operatorname{deg}(P)=\rho(f)=\rho(A) .
$$

Note that we can assume $\operatorname{deg}(R) \geq \operatorname{deg}(P)$ here, and then $\rho(A)=\rho(B) \geq \rho(f)$.

We will make frequent use of the fact that the derivative of an exponential polynomial $f$ is also an exponential polynomial and has the same leading coefficients as $f$.

Lemma 3.3. Let $f$ be an exponential polynomial of the (normalized) form

$$
f(z)=F_{0}(z)+F_{1}(z) e^{\lambda_{1} z^{q}}+\cdots+F_{m}(z) e^{\lambda_{m} z^{q}} .
$$

Then $f^{\prime}$ is also an exponential polynomial of order $q$, has precisely the same leading coefficients $\lambda_{1}, \ldots, \lambda_{m}$ as $f$, and $h_{f^{\prime}}(\theta) \leq h_{f}(\theta)$ holds for every $\theta \in \mathbf{R}$. If the strict inequality $h_{f^{\prime}}(\theta)<h_{f}(\theta)$ holds for some $\theta=\theta_{0}$, then $h_{f}\left(\theta_{0}\right)=0$.

Proof. A simple differentiation in (3.1) yields

$$
f^{\prime}(z)=F_{0}^{\prime}(z)+G_{1}(z) e^{\lambda_{1} z^{q}}+\cdots+G_{m}(z) e^{\lambda_{m} z^{q}},
$$

where $G_{j}(z)=F_{j}^{\prime}(z)+q \lambda_{j} z^{q-1} F_{j}(z)$ for $j=1, \ldots, m$. If $G_{j_{0}}(z) \equiv 0$ for some index $j_{0}$, then $F_{j_{0}}(z)=c \exp \left(-\lambda_{j_{0}} z^{q}\right)$, which is a contradiction. Hence the coefficients $G_{j}(z)$ are either ordinary polynomials in $z$ or exponential polynomials of order $\leq q-1$, and none of them vanishes identically. This proves that $f^{\prime}$ is an exponential polynomial of order $q$, and has the same leading coefficients $\lambda_{1}, \ldots, \lambda_{m}$ as $f$.

The indicator of a sum of two functions is less than or equal to the larger of the indicators:

$$
h_{g_{1}+g_{2}}(\theta) \leq \max \left\{h_{g_{1}}(\theta), h_{g_{2}}(\theta)\right\},
$$

see $[14$, p. 52]. If, for some value of $\theta$, the indicators of the two summands are not equal, then equality holds above. Since the leading coefficients $\lambda_{1}, \ldots, \lambda_{m}$ are pairwise distinct, the exponential terms $e^{\lambda_{1} z^{q}}, \ldots, e^{\lambda_{m} z^{q}}$ have distinct indicators. Thus

$$
h_{f}(\theta)= \begin{cases}\max _{0 \leq j \leq m} \Re\left(\lambda_{j} e^{i q \theta}\right), & F_{0}(z) \neq 0, \\ \max _{1 \leq j \leq m} \Re\left(\lambda_{j} e^{i q \theta}\right), & F_{0}(z) \equiv 0,\end{cases}
$$


and also

$$
h_{f^{\prime}}(\theta)= \begin{cases}\max _{0 \leq j \leq m} \Re\left(\lambda_{j} e^{i q \theta}\right), & F_{0}^{\prime}(z) \not \equiv 0, \\ \max _{1 \leq j \leq m} \Re\left(\lambda_{j} e^{i q \theta}\right), & F_{0}^{\prime}(z) \equiv 0,\end{cases}
$$

where $\lambda_{0}=0$. This yields $h_{f^{\prime}}(\theta)=h_{f}(\theta)$ except possibly in the case when $F_{0}(z)$ is a function for which $F_{0}^{\prime}(z) \equiv 0$ and $F_{0}(z) \not \equiv 0$, that is, when $F_{0}(z) \not \equiv 0$ is a constant function. In this case and only in this case it is possible that $h_{f^{\prime}}(\theta)<h_{f}(\theta)$, and if so, then clearly $h_{f}(\theta)=0$.

Next we will focus on the case $\rho(B)<\rho(A)=\rho(f)<\infty$, where $A(z)$ and $f$ are exponential polynomials.

Theorem 3.4. Let $A(z)$ be an exponential polynomial

$$
A(z)=K_{0}(z)+K_{1}(z) e^{\mu_{1} z^{q}}+\cdots+K_{l}(z) e^{\mu_{l} z^{q}},
$$

and let $B(z)$ be entire such that $\rho(B)<\rho(A)$. Suppose that (1.1) has a nontrivial solution $f$ of the form (3.1). Define $P=\left\{\theta \in[-\pi, \pi): h_{A}(\theta)>0\right\}, N=\{\theta \in$ $\left.[-\pi, \pi): h_{A}(\theta)<0\right\}$ and $Z=\left\{\theta \in[-\pi, \pi): h_{A}(\theta)=0\right\}$. Then the following assertions hold.

(1) The set $P$ has positive measure and $F_{0}(z) \equiv F_{0} \in \mathbf{C} \backslash\{0\}$.

(2) We have $h_{f^{\prime}}(\theta)=-h_{A}(\theta)$ and $h_{f}(\theta)=0$ for every $\theta \in P$.

(3) If $\rho\left(A f^{\prime}\right)<q$, then $B(z)$ and $A(z) f^{\prime}(z)$ are constant functions, and $q=1$.

(4) If $\rho\left(A f^{\prime}\right)=q$, then $h_{A}(\theta)=h_{f}(\theta)-h_{f^{\prime}}(\theta)$ for every $\theta, N=\emptyset$ and $Z$ has positive measure.

Remark 3.5. Suppose that $g$ is meromorphic in $\mathbf{C}$ and of finite order $\rho$. If $g$ has finitely many poles, then the Phragmén-Lindelöf indicator function $h_{g}(\theta)$ is welldefined for every $\theta$. Recall that the poles of a meromorphic function of finite order can be enclosed in a collection of discs known as an $R$-set, and that an arbitrary ray $\arg (z)=\theta$ meets infinitely many discs of an $R$-set in a set of measure zero, see [12, Chapter 5]. Therefore, even if $g$ has infinitely many poles, its indicator $h_{g}(\theta)$ is well-defined for almost all $\theta$.

Proof of Theorem 3.4. (1) We observe that if $h_{A}(\theta) \leq 0$ for all $\theta$, then the exponential polynomial $A(z)$ is of minimal type, which violates (1.6). Hence there exists a $\theta_{0}$ such that $h_{A}\left(\theta_{0}\right)>0$, so that $P \neq \emptyset$. By continuity of $h_{A}$, the set $P$ must have positive measure. Using (2.4) in (2.3), we deduce that $0<h_{A}(\theta) \leq h_{f / f^{\prime}}(\theta)$ for almost every $\theta \in P$. This is possible only if $h_{f^{\prime}}(\theta)<h_{f}(\theta)$. Finally, by Lemma 3.3 and by continuity, we have $h_{f^{\prime}}(\theta)<h_{f}(\theta)=0$ for every $\theta \in P$. In addition, $F_{0}(z) \not \equiv 0$ must be a constant function.

(2) The proof of Part (1) shows that $h_{f^{\prime}}(\theta)<h_{f}(\theta)=0$ for every $\theta \in P$. Hence $h_{A}(\theta) \leq-h_{f^{\prime}}(\theta)$ by $(2.3)$, while $-h_{f^{\prime}}(\theta) \leq h_{A}(\theta)$ for almost every $\theta \in P$ by

$$
\left|\frac{f(z)}{f^{\prime}(z)}\right| \leq\left(\left|\frac{f^{\prime \prime}(z)}{f^{\prime}(z)}\right|+|A(z)|\right)|B(z)|^{-1}
$$

By continuity again, we have $-h_{f^{\prime}}(\theta) \leq h_{A}(\theta)$ for every $\theta \in P$. This proves $h_{f^{\prime}}(\theta)=$ $-h_{A}(\theta)$ for every $\theta \in P$.

(3) The assumption $\rho\left(A f^{\prime}\right)<q$ implies that the convergence exponents of the zeros of $A(z)$ and of $f^{\prime}$ must both be $<q$. Then, by (1.7) and (1.8), we have $K_{0}(z) \equiv 0$ 
and $m=1=l$. Moreover, $\mu_{1}+\lambda_{1}=0$ must hold, for otherwise $\rho\left(A f^{\prime}\right)=q$. Therefore

$$
A(z) f^{\prime}(z)=G(z) K_{1}(z)
$$

where $G(z)=F_{1}^{\prime}(z)+q \lambda_{1} z^{q-1} F_{1}(z) \not \equiv 0$ by the proof of Lemma 3.3. Recalling Part (1), we have now proved that $A(z)=K_{1}(z) e^{-\lambda_{1} z^{q}}$ and $f(z)=F_{0}+F_{1}(z) e^{\lambda_{1} z^{q}}$. Similarly as in (3.2), a simple differentiation yields $f^{\prime \prime}(z)=H(z) e^{\lambda_{1} z^{q}}$, where $H(z)=$ $G^{\prime}(z)+q \lambda_{1} z^{q-1} G(z) \not \equiv 0$ is either an exponential polynomial of order $<q$ or an ordinary polynomial in $z$. A simple substitution to (1.1) yields

$$
B(z) F_{0}+K_{1}(z) G(z)+\left(B(z) F_{1}(z)+H(z)\right) e^{\lambda_{1} z^{q}}=0 .
$$

It follows by elementary exponential polynomial algebra [25, p. 77] that the coefficients in this equation must vanish, which allows us to write

$$
B(z)=-\frac{K_{1}(z) G(z)}{F_{0}}=-\frac{H(z)}{F_{1}(z)} .
$$

Next, we write

$$
K_{1}(z) F_{1}(z)=F_{0} \frac{H(z)}{G(z)}=F_{0}\left(\frac{G^{\prime}(z)}{G(z)}+q \lambda_{1} z^{q-1}\right) .
$$

Since the left-hand side of this equation is analytic, the function $G(z)$ cannot have any zeros. Hence $G(z)=e^{P(z)}$, where $P(z)$ is a polynomial such that $\operatorname{deg}(P)<q$. However, the primitive of

$$
f^{\prime}(z)=G(z) e^{\lambda_{1} z^{q}}=e^{\lambda_{1} z^{q}+P(z)}
$$

is supposed to be an exponential polynomial, and this is possible only if $q=1$ and $P(z)$ is a constant function. Therefore $G(z)$ and $F_{1}(z)$ are constant functions, and, a fortiori, $H(z), K_{1}(z)$ and $B(z)$ are constant functions. Finally, $A(z) f^{\prime}(z)$ is a constant function by (3.5).

(4) Since $A(z)$ and $f^{\prime}$ are both of c.r.g. by Lemmas 1.3 and 3.3, it follows by [17, p. 39] and the assumption $\rho\left(A f^{\prime}\right)=q$ that

$$
h_{A f^{\prime}}(\theta)=h_{A}(\theta)+h_{f^{\prime}}(\theta) .
$$

Making use of the inequality $\left|A(z) f^{\prime}\right| \leq|B(z) f|+\left|f^{\prime \prime}\right|$ and Lemma 3.3, we get

$$
h_{A f^{\prime}}(\theta) \leq \max \left\{h_{B f}(\theta), h_{f^{\prime \prime}}(\theta)\right\}=\max \left\{h_{f}(\theta), h_{f^{\prime \prime}}(\theta)\right\}=h_{f}(\theta) .
$$

This together with (3.6) yields $h_{A}(\theta) \leq h_{f}(\theta)-h_{f^{\prime}}(\theta)$ for every $\theta$. Writing (3.4) in the form

$$
|f(z)| \leq\left(\left|\frac{f^{\prime \prime}(z)}{f^{\prime}(z)}\right|+|A(z)|\right)|B(z)|^{-1}\left|f^{\prime}(z)\right|,
$$

we conclude the reverse inequality $h_{A}(\theta) \geq h_{f}(\theta)-h_{f^{\prime}}(\theta)$ for almost every $\theta$, and, by continuity, for every $\theta$. If $\theta \in N$, then Lemma 3.3 yields

$$
0>h_{A}(\theta)=h_{f}(\theta)-h_{f^{\prime}}(\theta) \geq 0,
$$

which is a contradiction. Hence $N=\emptyset$, and, a fortiori, $m(Z) \geq 1 /\left(21(q+1)^{2}\right)>0$ by Theorem 2.5 and the assumption that $\rho(A)=q=\rho(f)$.

If $f$ is an exponential polynomial solution of (1.1), where $A(z), B(z)$ are entire, then $\rho(A) \geq \rho(B)$. Theorem 3.4 provides many properties of the functions $A(z)$ and $f$ in the case when they are exponential polynomials with $\rho(A)>\rho(B)$. 
In the next result the functions $A(z), B(z), f$ are all exponential polynomials. If $\rho(A)=\rho(B)$, then $C\left(\operatorname{co}\left(W_{0}^{A}\right)\right) \geq C\left(\operatorname{co}\left(W_{0}^{B}\right)\right)$ must hold by (1.2) and (1.6). Here $W^{g}$ is the set of complex conjugates of the leading coefficients of the exponential polynomial $g$, and $W_{0}^{g}=W^{g} \cup\{0\}$. Examples 1.1 and 3.1 show that equality or strict inequality in $C\left(\operatorname{co}\left(W_{0}^{A}\right)\right) \geq C\left(\operatorname{co}\left(W_{0}^{B}\right)\right)$ may occur. The next tasks are to find when equality occurs, and the order of $f$ in relation to that of $A(z)$.

Theorem 3.6. Let $A(z)$ and $B(z)$ be exponential polynomials. Assume that (1.1) possesses an exponential polynomial solution $f$ of the form (3.1).

(1) Suppose that one of the following holds:

(i) $\rho(f)<\rho(A)$;

(ii) $\rho(f)=\rho(A)$ and either $F_{0}(z) \equiv 0$ or $F_{0}^{\prime}(z) \not \equiv 0$.

Then $\rho(A)=\rho(B)$ and $C\left(\operatorname{co}\left(W_{0}^{A}\right)\right)=C\left(\operatorname{co}\left(W_{0}^{B}\right)\right)$. Moreover, $h_{A}(\theta)=h_{B}(\theta)$ for every $\theta$ from which at least one of $h_{A}(\theta), h_{B}(\theta)$ is nonnegative.

(2) If $\rho(f) \geq \rho(A)+1$, then $F_{0}(z) \equiv 0, m=1, \lambda(f)<\rho(f), \rho(A)=\rho(B)$ and $C\left(\operatorname{co}\left(W_{0}^{A}\right)\right)=C\left(\operatorname{co}\left(W_{0}^{B}\right)\right)$.

Proof. (1) Recall that by differentiating $f$, we get (3.2). Similarly,

$$
f^{\prime \prime}(z)=F_{0}^{\prime \prime}(z)+H_{1}(z) e^{\lambda_{1} z^{q}}+\cdots+H_{m}(z) e^{\lambda_{m} z^{q}},
$$

where the coefficients $H_{j}(z)=G_{j}^{\prime}(z)+q \lambda_{j} z^{q-1} G_{j}(z) \not \equiv 0$ are again either ordinary polynomials in $z$ or exponential polynomials of order $\leq q-1$.

(i) Suppose first that $\rho(f)=q<\rho(A)$. Then (2.3) and (1.6) yield

$$
T(r, A) \leq T(r, B)+O\left(r^{q}\right)=T(r, B)+o(T(r, A)) .
$$

Combining this with (1.2), we obtain $\rho(A)=\rho(B)$. Moreover, $C\left(\operatorname{co}\left(W_{0}^{A}\right)\right)=$ $C\left(\operatorname{co}\left(W_{0}^{B}\right)\right)$ by $(1.6)$. Using $(2.3)$, we conclude that

$$
\log ^{+}\left|A\left(r e^{i \theta}\right)\right| \leq \log ^{+}\left|B\left(r e^{i \theta}\right)\right|+\log ^{+}\left|\frac{f^{\prime \prime}\left(r e^{i \theta}\right)}{f^{\prime}\left(r e^{i \theta}\right)}\right|+\log ^{+}\left|\frac{f\left(r e^{i \theta}\right)}{f^{\prime}\left(r e^{i \theta}\right)}\right| .
$$

In general, the indicator function $h_{g}$ of an exponential polynomial $g$ of order $q$ satisfies

$$
\log \left|g\left(r e^{i \theta}\right)\right|=\left(h_{g}(\theta)+o(1)\right) r^{q}
$$

outside of an exceptional set $E_{g}(r) \subset[-\pi, \pi)$ of $\theta$ 's whose Lebesgue measure satisfies $m\left(E_{g}(r)\right) \rightarrow 0$ as $r \rightarrow \infty$. This is apparent by the proof of [18, Satz 4], and has also been stated in [19, p. 462]. Divide (3.8) by $r^{\rho(A)}$ and let $r \rightarrow \infty$. By applying (3.9) to (3.8), we then conclude that

$$
h_{A}(\theta) \leq \max \left\{0, h_{B}(\theta)\right\}
$$

for every $\theta \in[-\pi, \pi)$ outside of a set of linear measure zero. Indeed, if $h_{A}(\theta) \leq 0$, then (3.10) is obviously true, while if $h_{A}(\theta)>0$, then $h_{B}(\theta)>0$ by (3.8) because $f, f^{\prime}, f^{\prime \prime}$ are all exponential polynomials of order $<\rho(A)$. By continuity, (3.10) holds for every $\theta$. The remaining assertion in (i) is then a simple consequence of (2.1) and (3.10).

(ii) Suppose then that $\rho(f)=q=\rho(A)$ and either $F_{0}(z) \equiv 0$ or $F_{0}^{\prime}(z) \not \equiv 0$. By the proof of Lemma 3.3, we conclude that the functions $f$ and $f^{\prime}$ have the same indicators. Now, applying $[19$, Satz $1(\mathrm{~b})]$ to $w=f / f^{\prime}$ gives us

$$
m\left(r, \frac{f}{f^{\prime}}\right)=o\left(r^{q}\right) .
$$


Using (2.3), (3.11) and then (1.2), (1.6), we get $\rho(A)=\rho(B)$ and $C\left(\operatorname{co}\left(W_{0}^{A}\right)\right)=$ $C\left(\operatorname{co}\left(W_{0}^{B}\right)\right)$. Moreover, since $h_{f}(\theta)=h_{f^{\prime}}(\theta)$ for all $\theta$, we have

$$
\log \left|\frac{f\left(r e^{i \theta}\right)}{f^{\prime}\left(r e^{i \theta}\right)}\right|=o\left(r^{q}\right)
$$

outside of an exceptional set $E_{f}(r)$ such that $m\left(E_{g}(r)\right) \rightarrow 0$ as $r \rightarrow \infty$. Combining this with (3.8) and keeping in mind that $q=\rho(A)$, we have (3.10) for almost every $\theta$, and hence for every $\theta$. Recalling (2.1), the remaining assertion in (ii) follows.

(2) Suppose that $\rho(f) \geq \rho(A)+1$. Then substitute $f$ as well as its derivatives (3.2) and (3.7) into (1.1) for

$$
F_{0}^{\prime \prime}+A(z) F_{0}^{\prime}+B(z) F_{0}+\sum_{j=1}^{m}\left(H_{j}+A(z) G_{j}+B(z) F_{j}\right) e^{\lambda_{j} z^{q}}=0 .
$$

By the assumption $q \geq \rho(A)+1$ and by elementary exponential polynomial algebra, see [25, p. 77], it follows that

$$
F_{0}^{\prime \prime}+A(z) F_{0}^{\prime}+B(z) F_{0} \equiv 0
$$

and

$$
H_{j}(z)+A(z) G_{j}(z)+B(z) F_{j}(z) \equiv 0, \quad j=1, \ldots, m .
$$

By (3.13), $F_{0}$ is a finite-order solution of (1.1), and is clearly not a multiple of $f$. This contradicts Theorem 4.1 below, unless $F_{0}(z) \equiv 0$. To prove that $m=1$, define $g(z)=F_{1}(z) e^{\lambda_{1} z^{q}}$. Then $g^{\prime}(z)=G_{1}(z) e^{\lambda_{1} z^{q}}$ and $g^{\prime \prime}(z)=H_{1}(z) e^{\lambda_{1} z^{q}}$. Next we multiply (3.14) in the case $j=1$ by $e^{\lambda_{1} z^{q}}$, and find that $g$ is a subnormal solution of (1.1). If $m \geq 2$, then $g$ is also linearly independent of $f$, which contradicts Theorem 4.1. Hence $m=1$.

We have proved that $F_{0}(z) \equiv 0$ and $m=1$. This gives us $\lambda(f)<\rho(f)$. Now [6, Theorem 1] implies that $T(r, A)=T(r, B)+O(\log r)$ as $r \rightarrow \infty$ outside of a possible exceptional set of finite logarithmic measure. Hence $\rho(A)=\rho(B)$ and $C\left(\operatorname{co}\left(W_{0}^{A}\right)\right)=C\left(\operatorname{co}\left(W_{0}^{B}\right)\right)$.

Corollary 3.7. If $\rho(A)=\rho(B)$ and $C\left(\operatorname{co}\left(W_{0}^{A}\right)\right)>C\left(\operatorname{co}\left(W_{0}^{B}\right)\right)$ in Theorem 3.6, then $\rho(f)=\rho(A)$ and $F_{0}(z) \equiv c \in \mathbf{C} \backslash\{0\}$. The strict inequality is necessary.

Proof. If $\rho(f)<\rho(A)$ or if $\rho(f) \geq \rho(A)+1$, then we arrive at a contradiction with Part (1)(i) or Part (2), respectively. Hence $\rho(f)=\rho(A)$, and then Part (1)(ii) yields $F_{0}(z) \equiv c \in \mathbf{C} \backslash\{0\}$. The necessity of the strict inequality $C\left(\operatorname{co}\left(W_{0}^{A}\right)\right)>C\left(\operatorname{co}\left(W_{0}^{B}\right)\right)$ follows by Example 1.1, see also (4.6).

The sharpness of Theorem 3.6 is illustrated as follows.

Example 3.8. (1)(i) Exponential polynomial solutions $f$ with $\rho(f)<\rho(A)$ are possible: If $B(z)$ is an exponential polynomial of order at least two and $A(z)=$ $-B(z)-1$, then $f(z)=e^{z}$ solves (1.1).

(1)(ii) To see that $m \geq 2$ is possible in the case $\rho(f) \leq \rho(A)$, we recall the following example from [6, p. 427]: For a fixed $\beta \in \mathbf{C}$, the function $f(z)=e^{\beta z}+e^{(\beta-1) z}$ solves (1.1), where

$$
\begin{aligned}
& A(z)=e^{\beta z}+e^{(\beta-1) z}+1-2 \beta, \\
& B(z)=\beta^{2}-\beta+(1-\beta) e^{(\beta-1) z}-\beta e^{\beta z} .
\end{aligned}
$$


Note that for $\beta \in \mathbf{C} \backslash \mathbf{R}$ the convex hulls $\operatorname{co}\left(W_{0}^{A}\right), \operatorname{co}\left(W_{0}^{B}\right)$ and $\operatorname{co}\left(W_{0}^{f}\right)$ are polygons. If $F_{0}(z)$ is a non-zero constant function, then $C\left(\operatorname{co}\left(W_{0}^{A}\right)\right)$ and $C\left(\operatorname{co}\left(W_{0}^{B}\right)\right)$ can be distinct (see Example 3.1) or equal: If $A(z)=-e^{2 z}$ and $B(z)=e^{2 z}+e^{z}$, then $f(z)=e^{z}-1$ solves $(1.1)$.

(2) The equality $\rho(f)=\rho(A)+1$ is possible, as is seen by means of

$$
f(z)=e^{z^{2}}, \quad A(z)=e^{z}, \quad B(z)=-2 z e^{z}-4 z^{2}-2 .
$$

More generally, $\rho(f)=\rho(A)+n$ for any $n \in \mathbf{N}$ is possible:

$$
f(z)=e^{z^{n+1}}, A(z)=e^{z}, B(z)=-(n+1) z^{n} e^{z}-(n+1)^{2} z^{2 n}-n(n+1) z^{n-1} .
$$

Example 1.1 shows that the inequality $\rho(f) \geq \rho(A)+1$ does not have to hold even if all of $F_{0}(z) \equiv 0, m=1, \rho(A)=\rho(B)$ and $C\left(\operatorname{co}\left(W_{0}^{A}\right)\right)=C\left(\operatorname{co}\left(W_{0}^{B}\right)\right)$ are valid. Hence the implication in Part (2) is not an equivalence.

Remark 3.9. (1) It is known that the value zero is the only possible deficient value for an admissible finite order solution of (1.1). This is the case in Theorem 3.6(2), for example. The proof of the rational coefficient case can be found in [24, pp. 53-54], and the general case is proved in [12, p. 62].

(2) Let $f$ be any exponential polynomial of the form (3.1), a solution of (1.1) or not. If $0 \notin \operatorname{co}\left(W^{f}\right)$, then $F_{0}(z)$ is a deficient target function for $f$, i.e., 0 is a deficient value for $f-F_{0}$. To see this, we note that since $\operatorname{co}\left(W^{f}\right)$ is a compact set, we have $C\left(\operatorname{co}\left(W^{f}\right)\right)<C\left(\operatorname{co}\left(W_{0}^{f}\right)\right)$. Now (1.8) yields

$$
N\left(r, \frac{1}{f-F_{0}}\right)=\left(C\left(\operatorname{co}\left(W^{f}\right)\right)+o(1)\right) \frac{r^{q}}{2 \pi}
$$

so that $\delta\left(0, f-F_{0}\right)=1-C\left(\operatorname{co}\left(W^{f}\right)\right) / C\left(\operatorname{co}\left(W_{0}^{f}\right)\right)>0$.

Example 3.10. Let $0<\alpha<1, \beta=(1-\alpha)^{-1}$, and let $A(z), B(z)$ be given by (3.15). As observed in [6, p. 427], the solution $f(z)=e^{\beta z}+e^{(\beta-1) z}$ of (1.1) then satisfies $N(r, 0, f)=(1+o(1)) r / \pi$ and $T(r, f)=(1+o(1)) \beta r / \pi$, so that

$$
\delta(0, f)=1-\beta^{-1}=\alpha .
$$

This shows that the value zero can be deficient for $f$ in the case $\rho(f)=\rho(A)$. There are no other finite deficient values as we may use Theorem A to see that $N(r, c, f)=(1+o(1)) \beta r / \pi$ for any $c \in \mathbf{C} \backslash\{0\}$, and hence $\delta(c, f)=0$. Note that $\operatorname{co}(W)$ is the closed interval $[\beta-1, \beta] \not \supset 0$.

The condition $0 \notin \mathrm{co}\left(W^{f}\right)$ is necessary: If $\beta=1 / 2$ and $A(z), B(z)$ are given by (3.15), then $f(z)=2 \cosh (z / 2)$ solves (1.1) and has no deficient values. In this case $0 \in[-1 / 2,1 / 2]=\operatorname{co}\left(W^{f}\right)=\operatorname{co}\left(W^{A}\right)=\operatorname{co}\left(W^{B}\right)$.

If $0<\beta<1$ in (3.15), then $\operatorname{co}\left(W^{A}\right)=\operatorname{co}\left(W^{B}\right)=[\beta-1, \beta] \ni 0$. Next we state two more examples in which $\operatorname{co}\left(W^{A}\right)$ and $\operatorname{co}\left(W^{B}\right)$ include the origin. Note also that in these examples $A(z)$ has three exponential terms, which is more than in the previous examples.

Example 3.11. [2, Examples 1-2] If $A(z)=-\left(e^{3 z}+e^{2 z}+e^{-z}\right)$ and $B(z)=$ $-\left(e^{2 z}+e^{-z}\right)$, then $f(z)=e^{-z}+1$ solves (1.1). If $A(z)=e^{z}+e^{-3 z}+e^{-2 z}$ and $B(z)=-\left(e^{z}+e^{-2 z}\right)$, then $f(z)=e^{z}+1$ solves $(1.1)$. 


\section{Slow and fast solutions}

Suppose that $A(z)$ and $B(z)$ are entire such that at least one of them is transcendental. Following Frei [4] and Wittich [23], we call any solution $f \not \equiv 0$ of (1.1) satisfying

$$
\limsup _{r \rightarrow \infty} r^{-1} \log T(r, f)=0
$$

as subnormal. In particular, a finite order solution is subnormal. The name reflects the fact that the growth is slower than expected. Frei showed in [4] that if $C \neq 0$ is a constant, then

$$
f^{\prime \prime}+e^{-z} f^{\prime}+C f=0
$$

possesses a subnormal solution if and only if $C=-n^{2}$ for some positive integer $n$. Wittich [23] extended this result as follows: If $P_{1}(z)$ and $P_{2}(z) \not \equiv 0$ are polynomials in $z$, and if $f \not \equiv 0$ is a subnormal solution of

$$
f^{\prime \prime}+P_{1}\left(e^{z}\right) f^{\prime}+P_{2}\left(e^{z}\right) f=0,
$$

then $f$ must be of the form $f(z)=e^{c z}\left(a_{0}+a_{1} e^{z}+\cdots+a_{m} e^{m z}\right)$, where $m \geq 0$ is an integer and $c, a_{0}, a_{1}, \ldots, a_{m}$ are constants with $a_{0} a_{m} \neq 0$.

Given two nonconstant polynomials $P_{1}(x, y)$ and $P_{2}(x, y)$ in arguments $x$ and $y$, Urabe and Yang have proved [21] that of any two linearly independent solutions of

$$
f^{\prime \prime}+P_{1}\left(e^{z}, e^{-z}\right) f^{\prime}+P_{2}\left(e^{z}, e^{-z}\right) f=0
$$

at most one can be subnormal. Further considerations are due to GundersenSteinbart [7] and many others.

It seems that until now subnormality has been associated only in the case where the coefficient functions are polynomials in $e^{z}$ and/or in $e^{-z}$. In the next result $A(z)$ is an arbitrary exponential polynomial, and there are no restrictions for the entire coefficient $B(z)$.

Theorem 4.1. Let $A(z)$ and $B(z)$ be entire functions, $\alpha>1$ a constant, and let $f_{1}, f_{2}$ be linearly independent solutions of (1.1). Then there exists a constant $r_{0}=r_{0}(\alpha)>0$ such that for all $r \geq r_{0}$, we have

$$
T(r, A) \leq \max \left\{\log T\left(\alpha r, f_{1}\right), \log T\left(\alpha r, f_{2}\right)\right\}+O(\log r) .
$$

If $A(z)$ satisfies

$$
\limsup _{r \rightarrow \infty} r^{-1} T(r, A)>0,
$$

then at most one of $f_{1}, f_{2}$ can be subnormal. In particular, if $A(z)$ is an exponential polynomial

$$
A(z)=H_{0}(z)+H_{1}(z) e^{w_{1} z^{n}}+\cdots+H_{m}(z) e^{w_{m} z^{n}}
$$

with $\rho(A)=n \geq 1$, then (4.2) holds and (4.1) can be replaced with

$$
\left(C\left(\operatorname{co}\left(W_{0}\right)\right)+o(1)\right) \frac{r^{n}}{2 \pi} \leq \max \left\{\log T\left(r, f_{1}\right), \log T\left(r, f_{2}\right)\right\},
$$

where $W_{0}=\left\{\overline{w_{0}}, \ldots, \overline{w_{m}}\right\}$.

Proof. We deduce by [12, Proposition 1.4.8] that the Wronskian $W=W\left(f_{1}, f_{2}\right)=$ $f_{1} f_{2}^{\prime}-f_{1}^{\prime} f_{2}$ can be written as

$$
W\left(f_{1}, f_{2}\right)(z)=C \exp \left(-\int^{z} A(\zeta) d \zeta\right)
$$


where $C \neq 0$ is some constant. Let $\beta=\sqrt{\alpha}$. Using the logarithmic derivative estimate due to Gol'dberg-Grinshtein [3, Corollary 3.2.3], we obtain

$$
T(r, A)=m(r, A)=m\left(r, \frac{W^{\prime}}{W}\right) \leq \log T(\beta r, W)+O(\log r)
$$

Writing

we have

$$
W=f_{1} f_{2}\left(\frac{f_{2}^{\prime}}{f_{2}}-\frac{f_{1}^{\prime}}{f_{1}}\right)
$$

$$
T(r, W)=m(r, W) \leq(1+o(1)) T\left(r, f_{1}\right)+(1+o(1)) T\left(r, f_{2}\right)
$$

for all $r$ outside of a set $E \subset[0, \infty)$ of finite linear measure. Set $\sigma=\int_{E} d r$ and $r_{0}=(\sigma+1) /(\beta-1)$. Now by the proof of [12, Lemma 1.1.1], we have

$$
T(r, W) \leq 3 \max \left\{T\left(\beta r, f_{1}\right), T\left(\beta r, f_{2}\right)\right\}
$$

for all $r \geq r_{0}$. Since $\beta^{2}=\alpha$, we have proved (4.1). Assuming (4.2), suppose on the contrary to the assertion that $f_{1}, f_{2}$ are both subnormal. By (4.2) there exists a sequence $\left\{r_{n}\right\}$ of positive real numbers tending to infinity such that

$$
\lim _{n \rightarrow \infty} r_{n}^{-1} T\left(r_{n}, A\right)>0 .
$$

Now substituting $r=r_{n}$ in (4.1) and dividing (4.1) by $\alpha r_{n}$, we arrive at a contradiction as $n \rightarrow \infty$.

Suppose then that $A(z)$ is an exponential polynomial. Since

$$
T(r, A)=\left(C\left(\operatorname{co}\left(W_{0}\right)\right)+o(1)\right) \frac{r^{n}}{2 \pi}
$$

by (1.6), it follows that (4.2) is clearly valid. The reasoning above gives us

$$
\left(C\left(\operatorname{co}\left(W_{0}\right)\right)+o(1)\right) \frac{r^{n}}{2 \pi \alpha^{n}} \leq \max \left\{\log T\left(r, f_{1}\right), \log T\left(r, f_{2}\right)\right\}
$$

for all $r \geq \alpha r_{0}$. In the course of proof of [12, Lemma 1.1.1] we may in fact choose $\beta=\beta(r)=1+(\sigma+1) / r$ for $r \geq 1$. Then

$$
\alpha^{-n}=\beta^{-2 n}=1+o(1), \quad r \rightarrow \infty,
$$

and hence (4.5) yields (4.3).

Theorem 4.1 suggests that an exponential of an exponential polynomial might be a good candidate for an infinite order entire solution. We can find an illustrative example from [6, p. 420]: If $b \neq 0$ is a constant, then $f_{1}(z)=e^{z}$ and $f_{2}(z)=$ $\exp \left(z+b e^{-2 z}\right)$ are solutions of

$$
f^{\prime \prime}+2 b e^{-2 z} f^{\prime}-\left(1+2 b e^{-2 z}\right) f=0 .
$$

Example 4.2. Suppose that $A(z)$ is an exponential polynomial of the form

$$
A(z)=P_{0}(z)+P_{1}^{\prime}(z) e^{P_{1}(z)}+\cdots+P_{n}^{\prime}(z) e^{P_{n}(z)},
$$

where $P_{0}(z), P_{1}(z), \ldots, P_{n}(z)$ are polynomials. Then any primitive function $\int^{z} A(\zeta) d \zeta$ of $A(z)$ is an exponential polynomial also. Note that this is closely related to (4.4). Now, if $q(z)$ is any (exponential) polynomial, then $f(z)=\exp \left(q(z)-\int^{z} A(\zeta) d \zeta\right)$ is a solution of

$$
f^{\prime \prime}+A(z) f^{\prime}+\left(A^{\prime}(z)+q^{\prime}(z) A(z)-q^{\prime \prime}(z)-q^{\prime}(z)^{2}\right) f=0 .
$$


The special case $q(z)=z$ and $A(z)=2 b e^{-2 z}$ reduces to (4.6).

Example 4.3. Let $g_{1}$ and $g_{2}$ be exponential polynomials such that

$$
g_{1}^{\prime}(z)-g_{2}^{\prime}(z)=e^{a z+b}
$$

for some $a, b \in \mathbf{C}$. Then $f_{1}=e^{g_{1}}$ and $f_{2}=e^{g_{2}}$ are linearly independent solutions of (1.1), where

$$
\begin{aligned}
& A(z)=-a-2 g_{1}^{\prime}(z)+e^{a z+b}, \\
& B(z)=\left(a+g_{1}^{\prime}(z)\right) g_{2}^{\prime}(z)+\left(g_{1}^{\prime}(z)+g_{2}^{\prime}(z)\right) e^{a z+b}-g_{1}^{\prime \prime}(z) .
\end{aligned}
$$

This shows that each of the two functions in a given fundamental solution base can be an exponential of an exponential polynomial.

We have $\rho(A)=\rho(B)$ in (4.7). An exponential of an exponential polynomial can be a solution of (1.1) also in the cases $\rho(A)<\rho(B)$ and $\rho(A)>\rho(B)$ :

Example 4.4. The function $f(z)=\exp \left(e^{z}\right)$ solves the equations

$$
\begin{aligned}
f^{\prime \prime}+\left(z e^{-z}-e^{2 z}-e^{z}\right) f^{\prime}+z f & =0, \\
f^{\prime \prime}+z f^{\prime}-\left(e^{z}+e^{2 z}+z e^{z}\right) f & =0 .
\end{aligned}
$$

Next we recall a result due to Kwon on the hyper-order of solutions.

Theorem 4.5. [11] Let $A(z)$ and $B(z)$ be entire functions such that $\rho(A)<\rho(B)$ or $\rho(B)<\rho(A)<1 / 2$. Then any entire solution $f \not \equiv 0$ of (1.1) satisfies

$$
\limsup _{r \rightarrow \infty} \frac{\log \log T(r, f)}{\log r} \geq \max \{\rho(A), \rho(B)\} .
$$

In fact an equality in (4.8) holds by general growth estimates for solutions of linear differential equations [9].

Theorem 4.6. Let $A(z)$ and $B(z)$ be entire functions of finite order such that $\rho(A) \neq \rho(B)$. Let $f=e^{g}$ be a solution of (1.1), where $g$ is an exponential polynomial. Then (4.8) holds with an equality.

Proof. We only need to prove the inequality (4.8). If $\rho(A)<\rho(B)$, then we are done. Suppose that $\rho(A)>\rho(B)$. The proof is based on the fact that $f^{\prime} / f=g^{\prime}$, where $g^{\prime}$ is an exponential polynomial and hence satisfies

$$
T\left(r, g^{\prime}\right)=(1+o(1)) T(r, g), \quad r \rightarrow \infty,
$$

by the proof of Lemma 3.3. We have

$$
\frac{f^{\prime \prime}}{f^{\prime}}=\frac{f^{\prime \prime} / f}{f^{\prime} / f}=\frac{\left(f^{\prime} / f\right)^{\prime}+\left(f^{\prime} / f\right)^{2}}{f^{\prime} / f}=\frac{\left(f^{\prime} / f\right)^{\prime}}{f^{\prime} / f}+\frac{f^{\prime}}{f}
$$

and

$$
\begin{aligned}
m\left(r, f^{\prime \prime} / f^{\prime}\right) & \leq m\left(r, f^{\prime} / f\right)+S\left(r, f^{\prime} / f\right)=T(r, g)+S(r, g), \\
m\left(r, f^{\prime} / f\right) & \leq T\left(r, f^{\prime} / f\right)+O(1)=(1+o(1)) T(r, g) .
\end{aligned}
$$

From (2.3) it now follows that

$$
\begin{aligned}
T(r, A) & \leq T(r, B)+m\left(r, f^{\prime \prime} / f^{\prime}\right)+m\left(r, f / f^{\prime}\right)+O(1) \\
& =T(r, B)+2 T(r, g)+S(r, g),
\end{aligned}
$$


where we use Gol'dberg-Grinshtein's estimate to conclude that

$$
\begin{aligned}
T(r, g) & =(1+o(1)) T\left(r, g^{\prime}\right)=(1+o(1)) m\left(r, f^{\prime} / f\right) \\
& \leq 2 \log (2 T(r, f))+O(\log r) .
\end{aligned}
$$

This yields (4.8) in the case $\rho(A)>\rho(B)$.

\section{References}

[1] BoAs, R.: Entire functions. - Academic Press Inc., New York, 1954.

[2] Chen, Z.-X., and K. H. Shon: On subnormal solutions of second order linear periodic differential equations. - Sci. China Ser. A 50:6, 2007, 786-800.

[3] Cherry, W., and Z. YE: Nevanlinna's theory of value distribution. The second main theorem and its error terms. - Springer Monogr. Math., Springer-Verlag, Berlin, 2001.

[4] FREI, M.: Über die subnormalen Lösungen der Differentialgleichung $w^{\prime \prime}+e^{-z} w^{\prime}+$ konst. $\cdot w=0$. - Comment. Math. Helv. 36, 1962, 1-8.

[5] Gundersen, G.: Estimates for the logarithmic derivative of a meromorphic function, plus similar estimates. - J. London Math. Soc. 37:1, 1988, 88-104.

[6] Gundersen, G.: Finite order solutions of second order linear differential equations. - Trans. Amer. Math. Soc. 305:1, 1988, 415-429.

[7] Gundersen, G., and E. Steinbart: Subnormal solutions of second order linear differential equations with periodic coefficients. - Results Math. 25, 1994, 270-289.

[8] Havin, V. P., and N. K. Nikolski (editors): Linear and complex analysis. Problem book 3. Part II. - Lecture Notes in Math. 1574. Springer-Verlag, Berlin, 1994.

[9] Heittokangas, J., R. Korhonen, and J. RÄтtyä: Growth estimates for solutions of linear complex differential equations. - Ann. Acad. Sci. Fenn. Math. 29:1, 2004, 233-246.

[10] Hellerstein, S., J. Miles, and J. Rossi: On the growth of solutions of $f^{\prime \prime}+g f^{\prime}+h f=0$. - Trans. Amer. Math. Soc. 324:2, 1991, 693-706.

[11] KwON, K.-H.: On the growth of entire functions satisfying second order linear differential equations. - Bull. Korean Math. Soc. 33:3, 1996, 487-496.

[12] Laine, I.: Nevanlinna theory and complex differential equations. - Walter de Gruyter, BerlinNew York, 1993.

[13] LAine, I., and R. YANG: Finite order solutions of complex linear differential equations. Electron. J. Differential Equations 2004:65, 2004, 1-8.

[14] Levin, B. YA.: Distribution of zeros of entire functions. Revised edition. - Transl. Math. Monogr. 5, Amer. Math. Soc., Providence, R.I., 1980.

[15] Miles, J., and J. Rossi: Linear combinations of logarithmic derivatives of entire functions with applications to differential equations. - Pacific J. Math. 174, 1996, 195-214.

[16] Petrenko, V. P.: Entire curves. - Kharkov, 1984 (in Russian).

[17] Ronkin, L. I.: Functions of completely regular growth. - Math. Appl. (Soviet Ser.) 81, Kluwer Academic Publishers Group, Dordrecht, 1992.

[18] Steinmetz, N.: Zur Wertverteilung von Exponentialpolynomen. - Manuscripta Math. 26:1-2, 1978/79, 155-167.

[19] Steinmetz, N.: Zur Wertverteilung der Quotienten von Exponentialpolynomen. - Arch. Math. (Basel) 35:5, 1980, 461-470.

[20] Tsuj, M.: Potential theory in modern function theory. Reprinting of the 1959 original. Chelsea Publishing Co., New York, 1975. 
[21] Urabe, H., and C.-C. YAnG: On factorization of entire functions satisfying differential equations. - Kodai Math. J. 14, 1991, 123-133.

[22] Voorhoeve, M., A. J. Van der Poorten, and R. Tijdeman: On the number of zeros of certain functions. - Nederl. Akad. Wetensch. Proc. Ser. A 78, Indag. Math. 37:5, 1975, 407-416.

[23] Wiтtich, H.: Subnormale Lösungen der Differentialgleichung $w^{\prime \prime}+p\left(e^{z}\right) w^{\prime}+q\left(e^{z}\right) w=0$. Nagoya Math. J. 30, 1967, 29-37.

[24] Wiтtich, H.: Neuere Untersuchungen über eindeutige analytische Funktionen. Zweite, korrigierte Auflage. - Ergeb. Math. Grenzgeb. 8, Springer-Verlag, Berlin-New York, 1968.

[25] YAnG, C.-C., and H.-X. YI: Uniqueness theory of meromorphic functions. - Math. Appl. 557, Kluwer Academic Publishers Group, Dordrecht, 2003.

Received 24 October 2014 • Received 13 May 2015 • Accepted 17 May 2015 